\title{
Pyogenic liver abscesses derived from dental disease in an immunocompetent patient
}

\author{
Luis Sanches Fonseca, Patrícia de Deus Caixeirinho, \\ Maria do Céu Machado, Ana Vintém Oliveira, Marvin Oliveira, \\ Jorge Manuel Pinheiro
}

\begin{abstract}
Introduction: Pyogenic liver abscess of odontogenic origin is extremely rare. The authors report a case of pyogenic liver abscesses with oral origin caused by Prevotella denticola and Streptococcus anginosus, in an immunocompetent adult. Case Report: A 54-year-old healthy female was presented to our hospital with fever, pain in the right hypochondriac region, constipation, anorexia and history of toothache with gingival swelling. Abdominal X-ray presented showed signs of intestinal obstruction and computed tomography suggested two liver abscesses. The larger one, with $113 \mathrm{~mm}$, was drained under percutaneous ultrasound guidance. Abscess culture isolated Prevotella denticola and Streptococcus anginosus. Under antibiotic therapy, the most common causes for liver abscess were excluded. Dental evaluation found multiple septic foci. Root extractions, dental restorations and
\end{abstract}

Luis Sanches Fonseca1, Patrícia de Deus Caixeirinho", Maria do Céu Machado ${ }^{1}$, Ana Vintém Oliveira ${ }^{2}$, Marvin OIiveira $^{3}$, Jorge Manuel Pinheiro ${ }^{4}$

Affiliations: ${ }^{1}$ Resident in Stomatology, Department of Stomatology, Centro Hospitalar de Lisboa Central, Lisbon, Portugal; ${ }^{2}$ Consultant in Internal Medicine, Intensive Care Unit, Centro Hospitalar de Lisboa Central, Lisbon, Portugal; ${ }^{3}$ Resident in Clinical Pathology, Department of Clinical Pathology, Centro Hospitalar de Lisboa Central, Lisbon, Portugal; ${ }^{4}$ Consultant in Stomatology, Department of Stomatology, Centro Hospitalar de Lisboa Central, Lisbon, Portugal. Corresponding Author: Luis Sanches Fonseca, Rua José António Serrano - Hospital de S. José - Serviço de Estomatologia, 1150-199, Lisbon, Portugal; Email: luisfilipefonseca@outlook.pt

Received: 25 October 2017

Accepted: 13 December 2017

Published: 22 January 2018 periodontal treatments were performed. In this case, abscess culture result is highly suggestive of a dental origin, as species isolated are common oral pathogens. Along with the clinical findings, we are reassured about the original source of infection. Conclusion: This case illustrates the underestimated risk associated with untreated dental diseases. We believe oral infectious foci removal is imperative as primary care. Due to the increase of the cryptogenic liver abscess, we find a systematic dental evaluation is required for the etiological diagnosis of liver abscess.

Keywords: Dental focal infections, Liver abscess, Prevotella denticola, Streptococcus anginosus

\section{How to cite this article}

Fonseca LS, Caixeirinho PDD, Machado MDC, Oliveira AV, Oliveira M, Pinheiro JM. Pyogenic liver abscesses derived from dental disease in an immunocompetent patient. J Case Rep Images Dent 2018;4:1-5.

Article ID: 100024Zo7LF2018

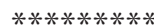

doi: 10.5348/Zo7-2018-24-CR-1

\section{INTRODUCTION}

Liver abscess is a rare, life-threatening disease, defined as a suppurated cavity with invasive microorganisms within the liver parenchyma $[1-3]$. The mortality rate is still elevated (ranging from $2-30 \%$ ) despite advances in antibiotic therapy and radiological diagnosis [3-5]. The etiology can be bacterial, parasitic (Entamoeba 
histolytica), mixed (pyogenic superinfection of a parasitic abscess) or, rarely, fungal [1, 3].

In Western countries, $80 \%$ of liver abscesses are bacterial [1].

Multiple diseases can cause pyogenic liver abscess (PLA) such as biliary tract disease, colonic disease, malignancy, recent gastric or enteric surgery, direct trauma or pancreatitis $[6,7]$. Hematogenous bacterial seeding is rarely reported [8]. Pyogenic liver abscess can also be cryptogenic [4-6]. Dental origin of PLA in an immunocompetent patient is extremely rare [6]. We report a case of PLA caused by Prevotella denticola and Streptococcus anginosus, in an immunocompetent adult.

\section{CASE REPORT}

A 54-year-old female was admitted to our hospital presenting fever, right hypochondriac pain, increased abdominal volume, constipation and anorexia with a few days of onset. The patient had history of retinitis pigmentosa and essential hypertension. She also had an intermittent toothache in the lower left quadrant associated with gingival swelling for the last four months, which improved with the spontaneous purulent drainage. The patient never sought dental evaluation.

Physical examination revealed tachycardia $110 \mathrm{bpm}$, fever $38.6^{\circ} \mathrm{C}$, abdominal distention and peritoneal reaction in the right hypochondrium. Laboratory results included normochromic, normocytic anemia with hemoglobin count of $9 \mathrm{~g} / \mathrm{dl}$ (normal 11.5-15.5 g/dl), neutrophilic leukocytosis, with a white blood cell count 20.7 $\times 10^{9} / \mathrm{L}$ (normal 4.5-11.0x10 $/ \mathrm{L}$ ), neutrophil count $17.3 \times 10^{9} / \mathrm{L}$ (normal $2.0-8.5 \times 10^{9} / \mathrm{L}$ ), elevated gammaglutamyl transferase levels $98 \mathrm{U} / \mathrm{L}$ (normal < $38 \mathrm{U} / \mathrm{L}$ ), alkaline phosphatase 137U/L (normal 30-120 U/L) and C-reactive protein levels $226.8 \mathrm{mg} / \mathrm{L}$ (normal $<5.0$ $\mathrm{mg} / \mathrm{L}$ ). Urinalysis was normal and blood cultures were made. Abdominal X-ray presented signs of intestinal obstruction and computed tomography (CT) scan showed two liver abscesses in the left lobe. The larger one measured $113 \mathrm{~mm}$ and the other $16 \mathrm{~mm}$ (Figure 1). Computed tomography scan also revealed colonic diverticular disease, with no signs of diverticulitis.

The patient underwent percutaneous ultrasound guided abscess drainage, left with a pigtail catheter. Abscess fluid was sent for bacterial culture. She was started on intravenous piperacillin/tazobactam $4 \mathrm{~g} / 0.5 \mathrm{~g}$ three times per day and metronidazole $500 \mathrm{mg}$ four times per day.

On day-3 of admission, she presented progressive dyspnea, oxygen desaturation (83\%), diminished vesicular murmur and respiratory acidosis. A weak response to acute treatment led to the admission into intensive care unit. X-ray revealed a bilateral pulmonary infiltrate. A thoracic and abdominal CT scans showed, also, pleural effusion in both lungs and a consolidation pattern in the
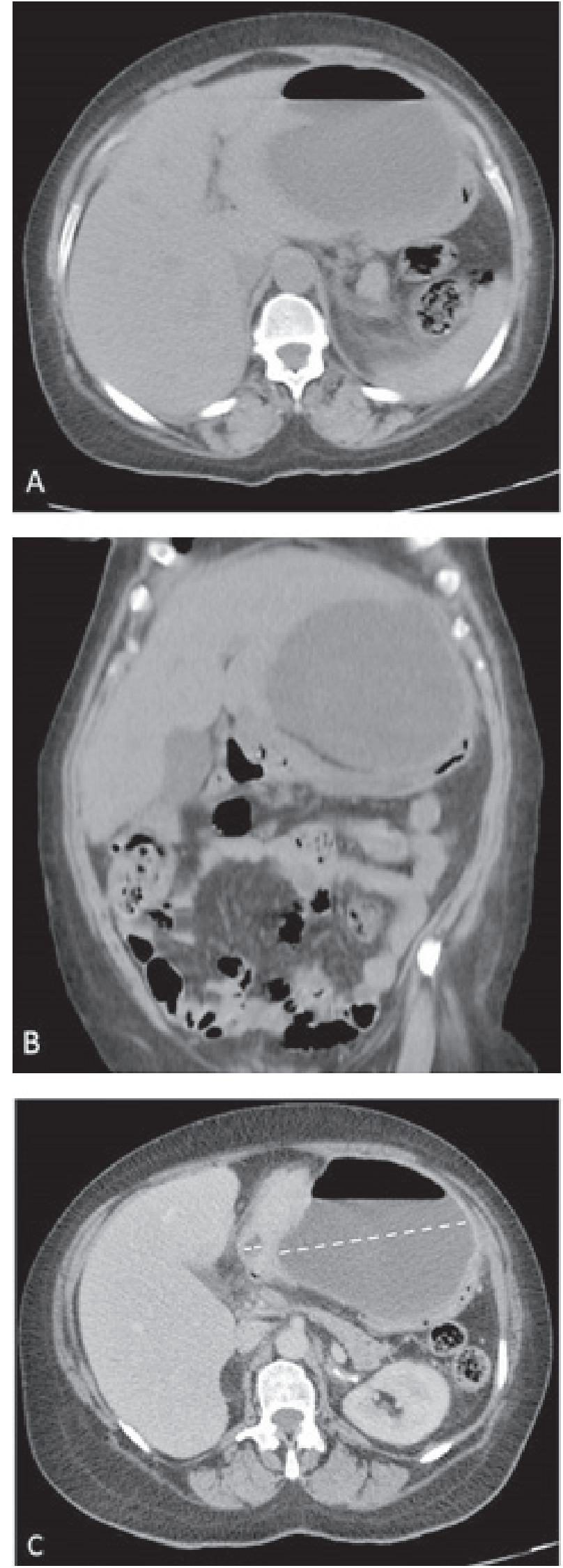

Figure 1: Initial abdominal CT: (A) Axial image and (B) Frontal image showing the larger abscess in the left hepatic lobe with an air-fluid level. (C) The compared diameters between abscesses (16 $\mathrm{mm}$ and $113 \mathrm{~mm}$ ). 
right lung. The liver abscesses were significantly reduced in the CT, with no signs of complications (Figure 2).

A thoracentesis was performed and the fluid turned out to be a transudate. We maintained a broad-spectrum antibiotics for the nosocomial pneumonia and the patient showed gradual, but steady, clinical and radiological improvement, without the need of mechanic ventilation.

Blood cultures were negative but the abscess cultures isolated Streptococcus anginosus penicillin-sensible and Prevotella denticola beta-lactamase negative.

Due to laboratory limitation to perform an antibiotic sensitivity test for anaerobes, a literature review of the antibiotic resistance profile of the Prevotella species was made [9-12]. Even though we knew the isolated species was beta-lactamase negative, our review led us to change the previous association of piperacillin/tazobactam to amoxicillin/clavulanic acid 1/o.2 g three times per day, maintaining metronidazole.

In spite of the nosocomial pneumonia, the patient made full recovery from the abscesses, confirmed in CT images.

During the etiological investigation of the PLA, the most common causes were excluded, such as biliary or colonic diseases. Due to the history of toothache in the lower left quadrant and possible odontogenic abscess, the patient was observed by the Department of Stomatology.

Physical examination showed many oral septic foci that could justify the bacteremia needed for the abscesses hematogenous seeding. The symptomatic posterior region of the left lower quadrant presented a residual root (first premolar) and an occlusal cavity in the first molar, presenting symptoms of reversible pulpitis, both with periodontal positive percussion (Figure 3). As for the remaining clinical and radiological evaluation, the patient had eight more decayed teeth and active periodontal pockets in the context of a generalized chronic periodontitis (Figure 4).

We eliminated all infectious foci with extraction of the residual root (the suspected source of infection), periodontal treatment and dental restorations were done.

\section{DISCUSSION}

Pyogenic liver abscess can be potentially lifethreatening, therefore, early recognition and timely intervention is key for a successful outcome [13]. Hematogenous bacterial seeding is reported to be rare, and there are few reports of PLA with a confirmed dental origin $[5,7,8]$.

In this case, the liver abscesses had an odontogenic cause. The most common causes were ruled out. Although with documented diverticular disease in the first CT scan, the patient had no clinical history and no signs or symptoms of diverticulitis or biliary disease. The hematogenous seeding abscesses are likely to be multiple, such as in our patient $[6,14]$. The chronic dental diseases with presumed recent periodontal abscess and the fact that oral pathogens were isolated in the hepatic fluid drainage confirms the odontogenic source of PLA.

The bacteria isolated from the pyogenic liver abscess were Streptococcus anginosus and Prevotella denticola. Streptococcus anginosus is one of three distinct species of the $S$. anginosus group, together with the $S$. intermedius and the $S$. constellatus $[7,15,16]$. This group is commonly a commensal of human mucous membrane, such as oral, pharynx, gastrointestinal and genitourinary, even though they can cause contiguous or metastatic infections in the presence of mucosal damage $[7,15]$. This group is frequently isolated in dental abscesses, as well as in brain or liver abscesses, in particular the $S$. intermedius [7, 15-18].

P. denticola is one of the pigmented Prevotella species, a frequent inhabitant of the oral flora as well, but also a predominant species in dental abscesses, with isolation
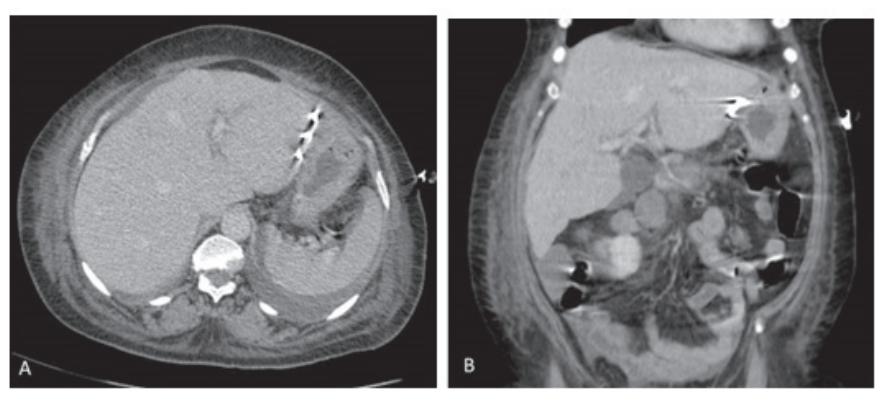

Figure 2: Third day abdominal computed tomography scan: (A) Axial image and (B) Frontal image showing successful fluid drainage with catheter in place and confirmed abscesses reductions.
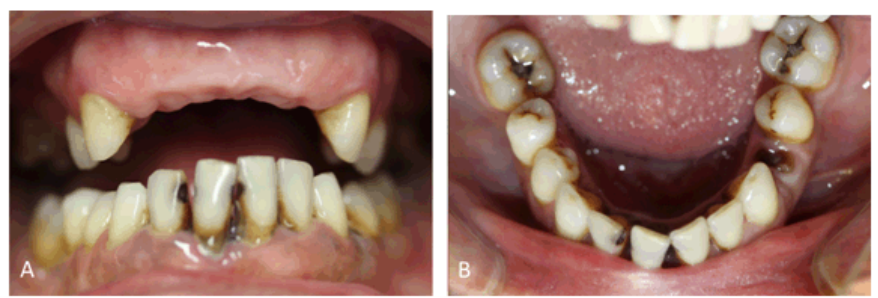

Figure 3: (A) Frontal intraoral photograph with decayed teeth and signs of periodontitis and (B) Occlusal intraoral photograph of the jaw showing both decayed molars and a residual root of the left mandibular first premolar.

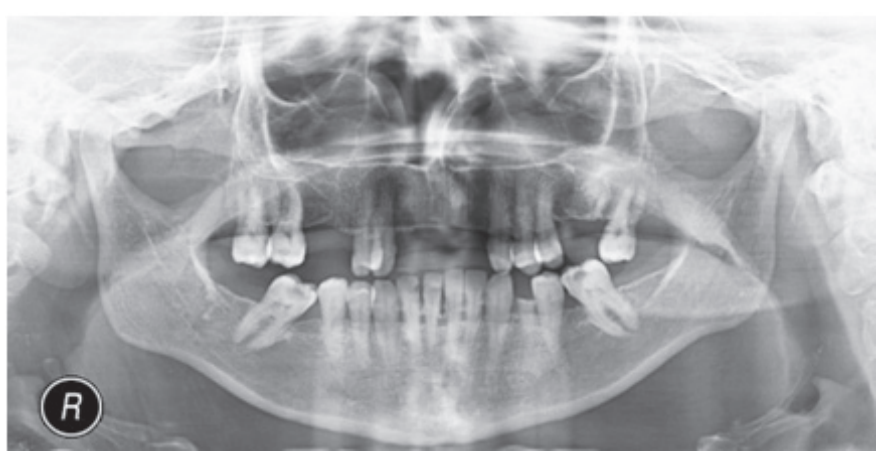

Figure 4: Panoramic radiograph showing the residual root of the left mandibular first premolar, multiple decayed teeth and diffuse vertical alveolar bone loss. 
rates of $31.37 \%[6,14,17]$. Not only isolated in dental and periodontal infections, $P$. denticola has been reported in superficial face and neck abscesses, endocarditis and brain abscesses [14].

As mentioned earlier, there are few case reports of PLA with an odontogenic cause. In fact, Osawa et al. [8], gathered a total of 13 PLA with dental origin in the literature until 2013. Only four of them were confirmed to be in immunocompromised patients. Some cases we found relevant were excluded from this search, such as a report of a liver abscess related to bacteremia following a routine dental cleaning [7].

Recent reports suggest an increase in frequency of cryptogenic liver abscesses, already with high rates [4, 6].

Concerning our case, we found only one case in literature of an odontogenic PLA associated with $S$. anginosus, but none associated with $P$. denticola. Lei et al. [6] reported an isolation of Prevotella species in their case, but did not specify which one.

\section{CONCLUSION}

This case is an excellent example of the underestimated risk associated with untreated dental diseases. Pyogenic liver abscess can occur from dental diseases bacteremia, even in immunocompetent patients, even though more rarely than other well known forms of metastatic odontogenic infections, as infectious endocarditis or artificial joints infection. We believe that a good oral heath and the elimination of oral infectious foci is imperative as primary care in both at risk or otherwise healthy patients. Due to the increase of the cryptogenic pyogenic liver abscesses, we find that a systematic dental evaluation is required for etiological diagnosis of all cryptogenic pyogenic liver abscesses, especially in patients with history of dental disease within weeks of the disease onset.-

\section{REFERENCES}

1. Lardière-Deguelte $\mathrm{S}$, Ragot $\mathrm{E}$, Amroun $\mathrm{K}$, et al. Hepatic abscess: Diagnosis and management. J Visc Surg 2015 Sep;152(4):231-43.

2. Gungor G, Biyik M, Polat H, Ciray H, Ozbek O, Demir A. Liver abscess after implantation of dental prosthesis. World J Hepatol 2012 Nov 27;4(11):31921.

3. Brook I, Frazier EH. Microbiology of liver and spleen abscesses. J Med Microbiol 1998 Dec;47(12):107580 .

4. Ohyama H, Nakasho K, Yamanegi K, et al. An unusual autopsy case of pyogenic liver abscess caused by periodontal bacteria. Jpn J Infect Dis 2009 Sep;62(5):381-3.

5. Yoneda M, Kato S, Mawatari H, et al. Liver abscess caused by periodontal bacterial infection with Fusobacterium necrophorum. Hepatol Res 2011 Feb;41(2):194-6.
6. Lei WY, Chang WH, Shih SC, Liu CJ, Shih CH. Pyogenic liver abscess with Prevotella species and Fusobacterium necrophorum as causative pathogens in an immunocompetent patient. $\mathrm{J}$ Formos Med Assoc 2009 Mar;108(3):253-7.

7. Livingston LV, Perez-Colon E. Streptococcus intermedius bacteremia and liver abscess following a routine dental cleaning. Case Rep Infect Dis 2014;2014:954046.

8. Osawa $\mathrm{Y}$, Isayama $\mathrm{H}$, Mizuno $\mathrm{S}$, et al. Two cases of liver abscesses derived from dental disease in patients with alcoholic chronic pancreatitis. Intern Med 2015;54(13):1623-5.

9. Kuriyama T, Williams DW, Yanagisawa M, et al. Antimicrobial susceptibility of 800 anaerobic isolates from patients with dentoalveolar infection to 13 oral antibiotics. Oral Microbiol Immunol 2007 Aug;22(4):285-8.

10. Maestre JR, Bascones A, Sánchez P, et al. Odontogenic bacteria in periodontal disease and resistance patterns to common antibiotics used as treatment and prophylaxis in odontology in Spain. Rev Esp Quimioter 2007 Mar;20(1):61-7.

11. Montagner F, Jacinto RC, Correa Signoretti FG, Scheffer de Mattos V, Grecca FS, Gomes BP. Betalactamic resistance profiles in Porphyromonas, Prevotella, and Parvimonas species isolated from acute endodontic infections. J Endod 2014 Mar;40(3):339-44.

12. Hecht DW. Prevalence of antibiotic resistance in anaerobic bacteria: Worrisome developments. Clin Infect Dis 2004 Jul 1;39(1):92-7.

13. Murarka S, Pranav F, Dandavate V. Pyogenic liver abscess secondary to disseminated Streptococcus anginosus from sigmoid diverticulitis. J Glob Infect Dis 2011 Jan;3(1):79-81.

14. Wu PC, Tu MS, Lin PH, Chen YS, Tsai HC. Prevotella brain abscesses and stroke following dental extraction in a young patient: A case report and review of the literature. Intern Med 2014;53(16):1881-7.

15. Giuliano S, Rubini G, Conte A, et al. Streptococcus anginosus group disseminated infection: Case report and review of literature. Infez Med 2012 Sep;20(3):145-54.

16. Neumayr A, Kubitz R, Bode JG, Bilk B, Häussinger D. Multiple liver abscesses with isolation of Streptococcus intermedius related to a pyogenic dental infection in an immuno-competent patient. Eur J Med Res 2010;15:319-22.

17. Robertson D, Smith AJ. The microbiology of the acute dental abscess. J Med Microbiol 2009 Feb;58(Pt 2):155-62.

18. Seppänen L, Lauhio A, Lindqvist C, Suuronen R, Rautemaa R. Analysis of systemic and local odontogenic infection complications requiring hospital care. J Infect 2008 Aug;57(2):116-22.

$$
* * * * * * * * *
$$

\section{Author Contributions}

Luis Sanches Fonseca - Substantial contributions to conception and design, Acquisition of data, Analysis and interpretation of data, Drafting the article, Revising 
it critically for important intellectual content, Final approval of the version to be published

Patrícia de Deus Caixeirinho - Substantial contribution to conception and design, Analysis and interpretation of data, Revising it critically for important intellectual content, Final approval of the version to be published Maria do Céu Machado - Analysis and interpretation of data, Revising it critically for important intellectual content, Final approval of the version to be published Ana Vintém de Oliveira - Analysis and interpretation of data, Revising it critically for important intellectual content, Final approval of the version to be published Marvin Oliveira - Analysis and interpretation of data, Revising it critically for important intellectual content, Final approval of the version to be published

Jorge Manuel Pinheiro - Analysis and interpretation of data, Revising it critically for important intellectual content, Final approval of the version to be published

\section{Guarantor of Submission}

The corresponding author is the guarantor of submission.

\section{Source of Support}

None

\section{Conflict of Interest}

Authors declare no conflict of interest.

\section{Copyright}

(C) 2018 Luis Sanches Fonseca et al. This article is distributed under the terms of Creative Commons Attribution License which permits unrestricted use, distribution and reproduction in any medium provided the original author(s) and original publisher are properly credited. Please see the copyright policy on the journal website for more information.
Access full text article on other devices

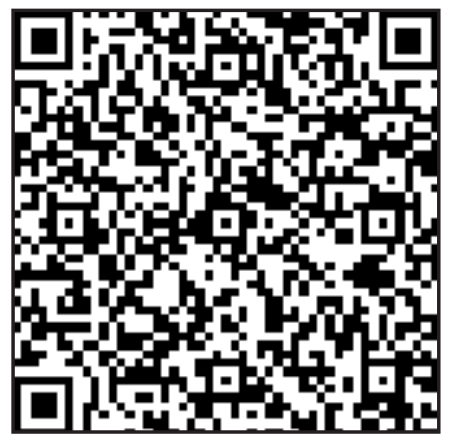

Access PDF of article on other devices

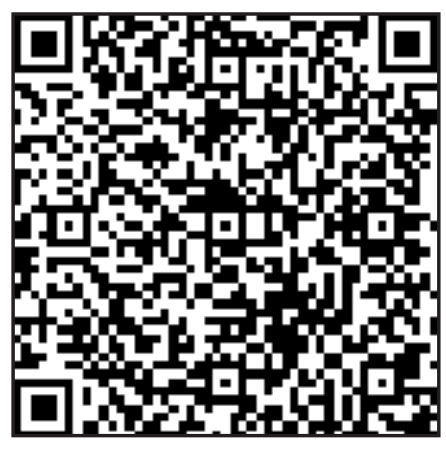

